\title{
Diffusion of Charged Colloidal Particles at Low Volume Fraction: Theoretical Model and Light Scattering Experiments
}

\author{
DIMITER N. PETSEV ${ }^{\prime}$ AND NIKOLAI D. DENKOV \\ Laboratory of Thermodynamics and Physico-chemical Hydrodynamics, Faculty of Chemistry, \\ University of Sofia, 1126 Sofia, Bulgaria
}

Received April 30, 1991; accepted August 21, 1991

\begin{abstract}
An appropriate mean force potential was utilized in Felderhof's Theory to derive simple analytical expressions for the concentration dependence of the collective and short time self diffusion coefficients, as well as for the sedimentation velocity of charged spherical particles. It is demonstrated theoretically that the osmotic virial and the Oseen hydrodynamic terms play a dominant role. To check the theoretical model, the dependence of the collective diffusion coefficient on the volume fraction of latex particles was experimentally studied. Dynamic light scattering was used at several different concentrations of electrolyte. It turns out that our experimental results, as well as the results of other authors, are in very good agreement with the proposed theoretical model. The results show that the increase of the electrolyte concentration leads to increase of the particle charge, but almost does not change the particle surface potential. A minimum in the dependence of the diffusion coefficient of a single particle on the ionic strength was also obtained. (c) 1992 Academic Press, Inc.
\end{abstract}

\section{INTRODUCTION}

The first step in the quantitative treatment of the Brownian diffusion was made by Einstein (1). He expressed the diffusion coefficient of spherical particles through the well known Stokes-Einstein relation,

$$
D_{\mathrm{SE}}=\frac{K T}{6 \pi \eta a}
$$

where $D_{\mathrm{SE}}$ is the diffusion coefficient, $K T$ is the thermal energy, $\eta$ is the solvent shear viscosity, and $a$ is the colloidal particle radius. This formula is valid for a single sphere, subjected to Brownian motion when no other spheres are present. The latter is a severe restriction, especially when the suspension is not infinitely diluted and/or long range interparticle forces are acting. Even the short range hard sphere interactions may affect the value of the diffusion coefficient, above a certain

\footnotetext{
${ }^{1}$ To whom correspondence should be addressed.
}

concentration of colloidal particles. Furthermore, such situations lead to a distinction of two types of diffusion coefficients: (i) self-diffusion coefficient, given by (2)

$$
D_{\mathrm{S}}=\frac{1}{3} \int_{0}^{\infty}\langle\mathbf{V}(0) \cdot \mathbf{V}(t)\rangle d t
$$

where $D_{\mathrm{S}}$ is the self diffusion coefficient, $\mathbf{V}$ is the velocity of a single particle, and $t$ is time; (ii) collective diffusion coefficient, which is the quantity that multiplies the concentration gradient in Fick's Law,

$$
\mathbf{j}=-D_{\mathrm{C}} \operatorname{grad} C .
$$

In Eq. [1.3], $\mathbf{j}$ is the particle flux, $D_{\mathrm{C}}$ is the collective diffusion coefficient, and $C$ is the particle concentration. When the suspension is infinitely diluted, the two coefficients $D_{\mathrm{S}}$ and $D_{\mathrm{C}}$ coincide. However, they may differ in the case of finite concentrations of Brownian particles. For example, if hard sphere interactions are present, $D_{\mathrm{S}}$ decreases with the particle 
concentration, while $D_{\mathrm{C}}$ increases, due to the excluded volume (see, e.g., (2)).

A further step in generalization of the Einstein theory was done by Batchelor, who combined hydrodynamical with statistical methods to develop a general approach to the Brownian motion of interacting spherical particles (3). Applying these methods to colloidal systems with hard sphere interactions, he obtained the first order correction of the collective diffusion coefficient with respect to the volume fraction of the particles $\phi(4)$,

$$
D_{\mathrm{C}}=D_{\mathrm{SE}}(1+\lambda \phi) \text {, }
$$

with $\lambda=1.45 . \lambda$ is the linear order coefficient, which in general depends on the interparticle interactions.

An approach, similar to that of Batchelor $(3,4)$, was developed by Felderhof and coworkers (5-9), who expressed $\lambda$ as a sum of integrals, depending on the pair correlation function, and two particle hydrodynamic interactions (6). The final result, $\lambda=1.454$, for hard spheres with no slip boundary conditions on their surfaces, coincides with that of Batchelor (see Eq. [1.4]).

In the case of charged particles, however, the situation is much more complex due to the necessity of using a screened Coulomb potential, in superposition with the hard sphere potential. A detailed review on this subject is given by Pusey and Tough (2), examining a great amount of theoretical and experimental investigations. An attempt for examination of such systems, both theoretically and experimentally, was made by Anderson et al. (10, 11). The experimental results in Ref. (11) showed a great increase of the collective diffusion coefficient with the decrease of the electrolyte concentration in the case of charged bovine serum albumin molecules. The theoretical approach in Ref. (10), however, was criticized by Felderhof (5) for the way in which the hydrodynamic interactions were taken into account. Another theoretical approach was proposed by Phillies $(12,13)$. He considered the hydrodynamic interactions in a manner, alternative to that of Felderhof and co-workers (5-9), which led to different results for the collective diffusion coefficient of hard spheres. This discrepancy, however, seems to be insignificant when suspensions of charged particles with low concentrations of electrolyte are considered (see Table I in Ref. (13)). Numerical calculations for the collective and self diffusion coefficients of charged colloidal spheres, based on the theory of Felderhof (5) and using a screened Coulomb type of potential, were performed by Ohtsuki and Okano (14). A simple analytical expression for the dependence of the collective diffusion coefficient on some parameters of the system was used by Belloni and Drifford (15). It accounts for the osmotic virial term and for the influence of the small ions but neglects the hydrodynamic interactions, which have been proved to be important in most cases $(14,16)$. Hence, no complete analytical formula was suggested to give an explicit functional relation between the concentration dependence of the collective diffusion coefficient and the surface potential of the particles, dielectric constant of the disperse medium, concentration of low molecular electrolyte, and particle radius. It is preferable to use simple expressions, similar to those for hard spheres (see Eq. [1.4]), rather than integrals, which need numerical evaluation. An alternative is to express $D_{\mathrm{C}}$ as a function of a parameter $\gamma$ which gives the ratio between an effective hard core repulsive radius $a_{\mathrm{eff}}$ and the actual hard sphere radius $a\left(a_{\mathrm{eff}}>a\right)$ ( see, e.g., (2)). A similar approach was used in a recent publication of Cichocki and Felderhof (16) for treatment of systems with interaction potentials, different from the hard sphere type. The effective hard core repulsive radius, introduced in (16), is not a quantity which can be measured independently. Besides, it has not been related in a simple way to the parameters which determine the actual electrostatic interactions.

The particle charge and the ionic strength of the suspension can influence the Brownian 
motion even of a single particle, because of deformation of the counterion atmosphere. The latter loses its spherical symmetry, thus creating an additional force, which decreases the particle velocity (17-19). This effect, however, is not due to the interparticle interactions and does not lead to dependence of the collective diffusion coefficient $D_{\mathrm{C}}$ on the particle volume fraction $\phi$. The polarization of the double layer can change the diffusion coefficient within 10-20\% (19), while the pair electrostatic interactions can increase the diffusivity several times (11). Similar is the situation when the viscosity of colloidal suspensions is examined. The secondary electroviscous effect, due to pair interactions, strongly dominates the primary one, due to deformation of the particle electrical double layer (20).

Using small ion-colloid particle coupled mode theory, Belloni and Drifford (15) showed that the small ions can also affect the particle concentration dependence of the collective diffusion coefficient. This effect is proportional to the ratio of the particle diffusion coefficient over that of the small ions and contributes much less significantly in comparison with the pair particle interactions.

Our aim in this study is to obtain the coefficient $\lambda$, defined by means of Eq. [1.4], as a simple function of some well defined quantities such as particle radius, charge and surface potential, screening parameter of the Coulomb interaction, dielectric and viscous properties of the disperse medium, and temperature.

The paper is organized as follows: Section 2 deals with the derivation of analytical expressions for the collective and the short time self diffusion coefficients of charged colloidal spheres as explicit functions of the suspension electrostatic properties; Section 3 presents the light scattering experiments for measurements of the diffusion coefficient of well defined latex suspensions; Section 4 is devoted to the comparison between our model and experimental results; a summary is given in Section 5; and some calculations are described in the Appendix.

\section{THEORY}

DERIVATION OF ANALYTICAL EXPRESSIONS FOR THE COLLECTIVE AND SELF DIFFUSION COEFFICIENTS OF CHARGED

\section{COlloidal SPHERES}

We follow the approach of Felderhof (5), which is based on the linear Stokes equations for an incompressible fluid:

$$
\eta \nabla^{2} \mathbf{v}=\nabla p, \quad \nabla \cdot \mathbf{v}=0 .
$$

Here $v$ is the velocity field and $p$ is the pressure.

The Brownian motion of the spherical colloidal particles is described with the $N$-particle Smoluchowski equation for the probability function $P_{N}$ in the configurational space (5),

$$
\begin{aligned}
\frac{\partial P_{N}}{\partial t}=\nabla_{N} \cdot \mathbf{D} \cdot[ & \nabla_{N} P_{N} \\
& \left.+\frac{1}{K T}\left(\nabla_{N} W_{N}\right) P_{N}\right],
\end{aligned}
$$

where $\mathbf{D}$ is the diffusion tensor and $W_{N}$ is the potential of interaction between $N$ particles. In accordance with Ref. (5), the following simplifications are assumed:

(i) the volume fraction of particles is low;

(ii) the interaction potential is pair-wise additive,

$$
\begin{aligned}
& W_{N}\left(r_{1}, r_{2}, \ldots, r_{N}\right) \\
&=\frac{1}{2} \sum_{i} \sum_{j \neq i} W\left(r_{i j}\right) ;
\end{aligned}
$$

(iii) the diffusion tensor is restricted to single particle, and pair contributions,

$$
\mathbf{D}=D \mathbf{I}+\sum_{i} \sum_{j}[\mathbf{A}(i, j)+\mathbf{B}(i, j)] .
$$

Here, the tensors $\mathbf{A}(i, j)$ and $\mathbf{B}(i, j)$ account for two particle hydrodynamic interactions. I is the unit tensor. (For more details one may refer to the original papers $(5,6))$;

(iv) the deviations of the pair correlation function $g(r)$ from its equilibrium shape are negligible; 
(v) the local density gradients, due to concentration fluctuations are small.

Under the above assumptions Felderhof derived (5)

$$
\frac{\partial \phi_{1}}{\partial t}=D_{\mathrm{C}} \nabla^{2} \phi_{1}
$$

where $\phi_{1}$ is a small perturbation of the average volume fraction $\phi . D_{\mathrm{C}}$ is the collective diffusion coefficient, and can be expressed as follows:

$$
\begin{aligned}
D_{\mathrm{C}} & =D_{0}(1+\lambda \phi) ; \\
\lambda & =\lambda_{\mathrm{V}}+\lambda_{\mathrm{O}}+\lambda_{\mathrm{A}}+\lambda_{\mathrm{S}}+\lambda_{\mathrm{D}} .
\end{aligned}
$$

$D_{0}$ is the diffusion coefficient of a single particle $(\phi \rightarrow 0)$. For charged colloidal particles $D_{0}$ may differ from the Stokes-Einstein value given by Eq. [1.1] (see, e.g., Refs. (17-19)). According to the theory of Felderhof (5), the coefficient $\lambda$ consists of five contribution terms:

$$
\lambda_{V}=-\frac{3}{a^{3}} \int_{0}^{\infty}[g(r)-1] r^{2} d r
$$

is the second osmotic virial coefficient;

$$
\lambda_{\circ}=\frac{3}{a^{2}} \int_{0}^{\infty}[g(r)-1] r d r
$$

is the Oseen hydrodynamic interaction term;

$$
\lambda_{\mathrm{A}}=\frac{3}{a^{3}} \int_{0}^{\infty}\left(\frac{9}{8} \frac{a^{6}}{r^{6}}-\frac{5}{4} \frac{a^{4}}{r^{4}}\right) g(r) r^{2} d r
$$

and

$$
\lambda_{\mathrm{S}}=\frac{75 a^{4}}{4} \int_{0}^{\infty} \frac{g(r)}{r^{5}} d r
$$

account for the short range hydrodynamic interactions; and

$$
\lambda_{\mathrm{D}}=1
$$

is a force dipole part. For hard spheres these quantities are estimated as follows:

$$
\begin{aligned}
& \lambda_{\mathrm{V}}=8, \quad \lambda_{\mathrm{O}}=-6, \quad \lambda_{\mathrm{A}}=-1.73, \\
& \lambda_{\mathrm{S}}=0.29, \quad \lambda_{\mathrm{D}}=1, \quad \text { or } \lambda=1.56
\end{aligned}
$$

It was shown recently (7-9) that the integral $\lambda_{\mathrm{A}}$ can be calculated with much better accuracy by involving higher order terms in the expansion of the two particle mobility functions, thus yielding $\lambda_{\mathrm{A}}=-1.83$ and $\lambda=1.45$ for hard spheres, which coincides with the result of Batchelor $(3,4)$. As we show below (see the comments after Eq. [2.33]), this specification of the hydrodynamic interactions affects only the hard sphere contribution, but can be completely neglected in the electrostatic repulsive one.

Equations [2.6]-[2.11] are the starting point for our treatment of charged colloidal systems. We write the radial distribution function $g(r)$ in the form:

$$
\begin{aligned}
g(r) & =\exp \left(-\frac{W_{\mathrm{HS}}}{K T}\right) \exp \left(-\frac{W_{\mathrm{EL}}}{K T}\right) \\
& \approx\left[\exp \left(-\frac{W_{\mathrm{HS}}}{K T}\right)\right]\left(1-\frac{W_{\mathrm{EL}}}{K T}\right)
\end{aligned}
$$

$W_{\mathrm{HS}}$ and $W_{\mathrm{EL}}$ are the hard sphere and electrostatic parts of the total potential of mean force. For a justification of this splitting of the interaction potential, see Ref. (21). Equation [2.13] implies the consideration of colloidal particles with low energy of pair electrostatic interaction, and allows one to split the integrals [2.7]-[2.10] into hard sphere and electrostatic parts. The potential of pair electrostatic interaction we use is the one derived by BeresfordSmith et al. (22),

$$
W_{\mathrm{EL}}=\frac{\left(z_{0} e\right)^{2}}{\epsilon} \frac{e^{2 \kappa a}}{(1+\kappa a)^{2}} \frac{e^{-\kappa r}}{r},
$$

where $z_{0}$ is the number of charges per colloidal particle, $e$ is the elementary charge, $\epsilon$ is the dielectric constant, $a$ is the particle radius, and $\kappa$ is the screening parameter. The original expression of Beresford-Smith et al. (22) includes a multiplier $(1+\phi)^{2}$. Since we are interested only in the first order correction of the collective diffusion coefficient $D_{\mathrm{C}}$ with respect to the volume fraction $\phi$ (which means zero order of the interaction potential), the 
higher order terms stemming from this multiplier can be omitted. The expression [2.14] was derived by means of statistical mechanical considerations using the correlation function formalism. It should be noted that the linearized Poisson-Boltzmann equation was used when deriving Eq. [2.14], in combination with some specific closure approximations. This equation, in fact, resembles the known DebyeHuckel expression (21), but there are some very important differences. The screening parameter $\kappa$ accounts for the presence of counterions, besides the added low molecular electrolyte (see Ref. (22)):

$$
\kappa^{2}=\frac{4 \pi e^{2}}{\epsilon K T} \sum_{i} n_{i} z_{i}^{2} .
$$

The summation in the right hand side is carried out over all low molecular ionic species in the suspension (added low molecular salt together with the free counterions dissociated from the colloidal particles). The corresponding concentration and number of charges per given ion are denoted by $n_{i}$ and $z_{i}$. This fact allows one to treat even suspensions with low concentration of added electrolyte, when the concentration of the counterions dissociated from the particles cannot be neglected. Another important property of the potential [2.14] is that it is ensemble averaged over the configurations of the remaining colloidal particles and the small ions, hence, it is a potential of mean force.

\subsection{Calculation of the Osmotic Virial Term $\lambda_{V}$}

Combining [2.7], [2.13], and [2.14] we obtain

$$
\begin{aligned}
& \lambda_{\mathrm{V}}=\frac{3}{a^{3}} \int_{0}^{2 a} r^{2} d r \\
& +\frac{3}{a^{3}} \int_{2 a}^{\infty} \frac{\left(z_{0} e\right)^{2}}{\epsilon K T} \frac{e^{2 \kappa a}}{(1+\kappa a)^{2}} e^{-\kappa r} r d r .
\end{aligned}
$$

The first integral in the right hand side of [2.16], denoted below by $\lambda_{\mathrm{V}}^{\mathrm{HS}}$, is responsible for the hard sphere contribution,

$$
\lambda_{\mathrm{V}}^{\mathrm{HS}}=8 \text {. }
$$

The second integral in Eq. [2.16] deals with the electrostatic interactions and is equal to

$$
\lambda_{V}^{\mathrm{EL}}=\frac{\left(z_{0} e\right)^{2}}{\epsilon K T a} \frac{(1+2 \kappa a)}{(1+\kappa a)^{2}} \frac{3}{(\kappa a)^{2}} .
$$

\subsection{Calculation of the Oseen Hydrodynamic Term $\lambda_{o}$}

Eqs. [2.8], [2.13], and [2.14] give

$$
\begin{array}{rl}
\lambda_{\mathrm{O}}=-\frac{3}{a^{2}} \int_{0}^{2 a} & r d r-\frac{3}{a^{2}} \int_{2 a}^{\infty} \frac{\left(z_{0} e\right)^{2}}{\epsilon K T} \\
& \times \frac{e^{2 \kappa a}}{(1+\kappa a)^{2}} e^{-\kappa r} d r .
\end{array}
$$

Again, the first integral gives the hard sphere part,

$$
\lambda_{O}^{\mathrm{HS}}=-6,
$$

and the second, the electrostatic contribution,

$$
\lambda_{\mathrm{O}}^{\mathrm{EL}}=-\frac{\left(z_{0} e\right)^{2}}{\epsilon K T a} \frac{1}{(1+\kappa a)^{2}} \frac{3}{(\kappa a)} .
$$

Equations [2.18] and [2.21] are obtained without imposing any restrictions on the value of $k a$.

We can write

$$
-\lambda_{V}^{\mathrm{EL}} / \lambda_{\mathrm{O}}^{\mathrm{EL}}=(2+1 / \kappa a)
$$

Hence, $\left|\lambda_{\mathrm{V}}^{\mathrm{EL}}\right|>\left|\lambda_{\mathrm{O}}^{\mathrm{EL}}\right|$ and for extremely small $\kappa a$ the osmotic virial term $\lambda_{\mathrm{V}}^{\mathrm{EL}}$ is the only term to be kept. This approximation is exploited in some theoretical models (15) and the interpretation of light scattering experiments with charged suspensions (2). However, in most cases $(14,16)$ the Oseen part $\lambda_{0}$ should be accounted for. It is shown below that the other hydrodynamic terms $\lambda_{\mathrm{A}}^{\mathrm{EL}}$ and $\lambda_{\mathrm{S}}^{\mathrm{EL}}$ are even less important. 
2.3. Calculation of the Short Range Term $\lambda_{A}$

In this case, Eqs. [2.9], [2.13], and [2.14] are used to yield

$$
\begin{aligned}
\lambda_{\mathrm{A}}= & \frac{3}{a^{3}} \int_{2 a}^{\infty}\left(\frac{9}{8} \frac{a^{6}}{r^{4}}-\frac{5}{4} \frac{a^{4}}{r^{2}}\right) d r \\
- & \frac{3}{a^{3}} \int_{2 a}^{\infty}\left(\frac{9}{8} \frac{a^{6}}{r^{4}}-\frac{5}{4} \frac{a^{4}}{r^{2}}\right) \\
& \quad \frac{\left(z_{0} e\right)^{2}}{\epsilon K T} \frac{e^{2 \kappa a}}{(1+\kappa a)^{2}} \frac{e^{-\kappa r}}{r} d r .
\end{aligned}
$$

The first integral accounts for the hard sphere interactions,

$$
\lambda_{\AA}^{\mathrm{HS}}=-1.73
$$

As we mentioned above, a more precise value $\lambda_{A}^{\mathrm{HS}}=-1.83$ was obtained recently (7-9). The second integral is the electrostatic term,

$$
\begin{aligned}
\lambda_{\AA}^{\mathrm{EL}} & =\frac{\left(z_{0} e\right)^{2}}{\epsilon K T a} \frac{e^{2 \kappa a}}{(1+\kappa a)^{2}} \\
\times & \times\left[-\frac{27}{128} E_{5}(2 \kappa a)+\frac{15}{16} E_{3}(2 \kappa a)\right],
\end{aligned}
$$

where $E_{n}(p)$ is an integral exponent of $n$th order, and is defined by $(23,24)$

$$
E_{n}(p)=\int_{1}^{\infty} \frac{e^{-s p}}{s^{n}} d s .
$$

It is not possible to solve analytically the integrals in [2.25] and [2.26] in the same way as for $\lambda_{\mathrm{V}}^{\mathrm{EL}}$ and $\lambda_{O}^{\mathrm{EL}}$. Still, an asymptotic expressions for the limiting cases $\kappa a \ll 1$, and $\kappa a \gg$ 1 can be derived (cf., Appendix and Refs. (23, 24)):

$$
\begin{array}{r}
\lambda_{\AA}^{\mathrm{EL}}=\frac{\left(z_{0} e\right)^{2}}{\epsilon K T a}\left[0.42-1.73 \kappa a+\mathrm{O}(\kappa a)^{2}\right] \\
\text { for } \kappa a \ll 1
\end{array}
$$

and

$$
\begin{array}{r}
\lambda_{\AA}^{\mathrm{EL}}=\frac{\left(z_{0} e\right)^{2}}{\epsilon K T a} \frac{0.363}{(\kappa a)^{3}}\left[1-\frac{3.21}{\kappa a}+\mathrm{O}(\kappa a)^{-2}\right] \\
\quad \text { for } \kappa a \gg 1 . \quad[2.28]
\end{array}
$$

The coefficient $\lambda_{A}^{\mathrm{EL}}$ is also related to the short time self diffusion coefficient $(2,16)$.

\subsection{Calculation of the Short Range Hydrodynamic Term $\lambda_{S}$}

In the same way as in the preceding cases we obtain

$$
\begin{aligned}
\lambda_{\mathrm{S}}=\frac{75}{4} a^{4} & \int_{2 a}^{\infty} \frac{d r}{r^{5}}-\frac{75}{4} a^{4} \frac{\left(z_{0} e\right)^{2}}{\epsilon K T} \\
& \times \frac{e^{2 \kappa a}}{(1-\kappa a)^{2}} \int_{2 a}^{\infty} \frac{e^{-\kappa r}}{r^{6}} d r,
\end{aligned}
$$

where we have again the hard sphere part

$$
\lambda_{S}^{\mathrm{HS}}=0.29
$$

and electrostatic part

$$
\begin{aligned}
& \lambda_{\mathrm{S}}^{\mathrm{EL}}=\frac{\left(z_{0} e\right)^{2}}{\epsilon K T a} \frac{e^{2 \kappa a}}{(1+\kappa a)^{2}} \\
& \times\left[-\frac{75}{128} E_{6}(2 \kappa a)\right] \text {. }
\end{aligned}
$$

The coefficient $\lambda_{\mathrm{S}}^{\mathrm{EL}}$ gives a negligible contribution to the collective diffusion coefficient (see the next subsection) and does not contribute to the short time self diffusion coefficient at all. It is a matter of simple mathematics to derive approximate expressions in the same manner as for Eqs. [2.27], and [2.28] (cf., Appendix and Refs. $(23,24)$ ).

\subsection{First Order Correction of the Diffusion Coefficients $D_{C}, D_{S}^{S}$, and Sedimentation Velocity $U$}

Equations [2.18] and [2.21] can be summed up to yield

$$
\lambda_{V}^{\mathrm{EL}}+\lambda_{\mathrm{O}}^{\mathrm{EL}}=\frac{\left(z_{0} e\right)^{2}}{\epsilon K T a} \frac{1}{(1+\kappa a)} \frac{3}{(\kappa a)^{2}} .
$$


Similarly, Eqs. [2.25] and [2.31] give

$$
\begin{array}{r}
\lambda_{\AA}^{\mathrm{EL}}+\lambda_{\mathrm{S}}^{\mathrm{EL}}=\frac{\left(z_{0} e\right)^{2}}{\epsilon K T a} \frac{e^{2 \kappa a}}{(1+\kappa a)^{2}} \\
\times\left[-\frac{27}{128} E_{5}(2 \kappa a)+\frac{15}{16} E_{3}(2 \kappa a)\right. \\
\left.-\frac{75}{128} E_{6}(2 \kappa a)\right] .
\end{array}
$$

The ratio $\left.\left(\lambda_{A}^{E L}+\lambda_{S}^{E L}\right) / \lambda_{V}^{E L}+\lambda_{O}^{E L}\right)$ is plotted in Fig. 1 as a function of $\kappa$. It is seen that this ratio passes through a maximum value at $\kappa a$ $\approx 5$, and tends to zero in the limit $\kappa a \rightarrow 0$. At large values of $k a$ the curve tends to a constant equal to 0.023 . The maximum of the ratio is 0.03 , which means that the main electrostatic contribution to the collective diffusion coefficient (see Eq. [2.6]) is due to the virial and the Oseen terms for all values of $k a$. The other terms, $\lambda_{\AA}^{\mathrm{EL}}$ and $\lambda_{\mathrm{S}}^{\mathrm{EL}}$, can be entirely neglected, at the cost of an error not exceeding $3 \%$. In spite of following another approach, Cichocki and Felderhof (16) also came to the same conclusion that only the virial and the Oseen contributions are important for the determination of the collective diffusion coefficient of charged Brownian spheres.

Combining Eqs. [2.6] and [2.32] we finally obtain a simple expression for the interaction parameter:

$$
\lambda=1.45+\frac{\left(z_{0} e\right)^{2}}{\epsilon K T a} \frac{1}{(1+\kappa a)} \frac{3}{(\kappa a)^{2}} .
$$

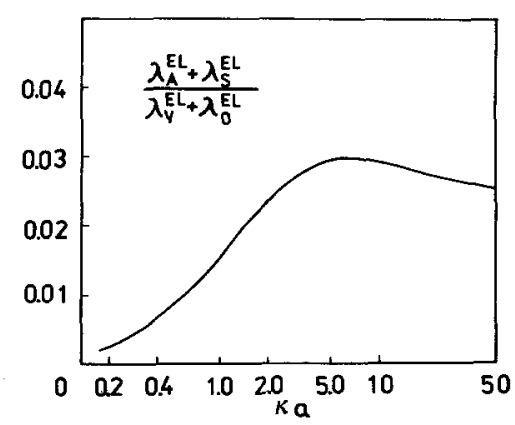
$\kappa a$.

FIG. 1. Plot of the ratio $\left(\lambda_{A}^{\mathrm{EL}}+\lambda_{S}^{\mathrm{EL}}\right) /\left(\lambda_{\mathrm{V}}^{\mathrm{EL}}+\lambda_{O}^{\mathrm{EL}}\right)$ vs.
The above results enable us to obtain in a similar manner a simple formula for the short time self diffusion coefficient $D_{\mathrm{S}}^{\mathrm{S}}$. Indeed, by using the formula $(2,16)$,

$$
D_{\mathrm{S}}^{\mathrm{S}}=D_{0}\left(1+\lambda_{\mathrm{A}} \phi\right),
$$

together with Eqs. [2.24] and [2.25], one obtains an expression for calculating $D_{\mathrm{S}}^{\mathrm{S}}$. For estimates, the asymptotic formulas [2.27] or [2.28] can be also used.

Another useful expression that can be obtained from our simple model is that for the sedimentation velocity of colloidal particles. Following the concept of Batchelor (3), and Felderhof (5), and taking into account our results above, we can write

$\frac{U}{U_{0}}=1$

$$
+\left(\lambda_{\mathrm{O}}^{\mathrm{HS}}+\lambda_{\mathrm{A}}^{\mathrm{HS}}+\lambda_{\mathrm{S}}^{\mathrm{HS}}+\lambda_{\mathrm{D}}+\lambda_{\mathrm{O}}^{\mathrm{EL}}\right) \phi[2.36]
$$
or

$$
\begin{aligned}
\frac{U}{U_{0}}= & \\
& -\left(6.54+\frac{\left(z_{0} e\right)^{2}}{\epsilon K T a} \frac{1}{(1+\kappa a)^{2}} \frac{3}{(\kappa a)}\right) \phi,
\end{aligned}
$$

where $U_{0}$ is the sedimentation velocity of an isolated particle and $U$ is the particle mean velocity in the presence of other particles. The terms $\lambda_{\mathrm{A}}^{\mathrm{EL}}$ and $\lambda_{\mathrm{S}}^{\mathrm{EL}}$ in Eq. [2.36] are neglected because their contribution is less than $3 \%$ (cf. Fig. 1).

Equation [2.34] is the basic result in our theoretical approach. The interaction parameter $\lambda$ is expressed as a function of the surface charge $\left(z_{0} e\right)$. However, it is more convenient in some cases to use an expression which depends on the surface potential of the particles $\Psi_{\text {S. }}$ Such expression is derived below. We start our considerations, using the expression for the total ion-colloid correlation function $h_{10}$, given by Beresford-Smith et al. (22),

$$
h_{10}=-\frac{z_{1} z_{0} e^{2}}{\epsilon K T} \frac{e^{\kappa a}}{(1+\kappa a)} \frac{e^{-\kappa r}}{r},
$$


where $z_{1}$ is the number of charges of the corresponding microion. Hence, the potential distribution around the colloidal particle is (21)

$$
\Psi(r)=\frac{z_{0} e}{\epsilon} \frac{e^{\kappa a}}{(1+\kappa a)} \frac{e^{-k r}}{r} .
$$

The surface potential of the particle is

$$
\Psi_{\mathrm{S}}=\frac{z_{0} e}{\epsilon a} \frac{1}{(1+\kappa a)} .
$$

Introducing [2.40] we obtain

$$
W_{\mathrm{EL}}=\Psi_{\mathrm{S}}^{2} \epsilon a^{2} e^{2 \kappa a} \frac{e^{-\kappa r}}{r},
$$

which in fact is the potential of mean force of Beresford-Smith et al. (22), expressed in terms of the surface potential $\Psi_{\mathrm{s}}$. Thus, for the interaction parameter $\lambda$ we get (cf., Eq. [2.34])

$$
\lambda=1.45+\frac{\Psi_{\mathrm{S}}^{2} \epsilon a}{K T}(1+\kappa a) \frac{3}{(\kappa a)^{2}} .
$$

When a system with a given constant charge is considered, according to Eq. [2.34], the electrostatic correction of $\lambda$ diverges for small $\kappa a$ as $(\kappa a)^{-2}$ and tends to zero for large $\kappa a$ as $(\kappa a)^{-3}$. When the real physical situation corresponds to a constant surface potential, Eq. [2.42] implies that the electrostatic correction of $\lambda$ diverges for small $\kappa a$ as $(\kappa a)^{-2}$ and tends to zero for large $\kappa a$ as $(\kappa a)^{-1}$. The experimental results, obtained in the next section, seem to favor the second case when the surface potential is constant. There might be other cases, however, where the situation is different.

We should like to emphasize that the asymptote at $\kappa a \rightarrow \infty$ gives properly the hard sphere result of Batchelor $(3,4)$ and Felderhof and co-workers (5-9). For real systems, however, the increase in $\kappa a$ leads to an increase of the importance of the Van der Waals attractive forces. Hence, they should be also included when $\kappa a$ is large. We are interested in charged systems at low concentrations of electrolyte, and the attractive interactions can be entirely neglected. For example, Corti and Degiorgio
(25) showed in their experiments with ionic micelles, that for $\kappa a<2.6$ the Van der Waals attractive contribution is less than few percents.

Another possible effect we are neglecting in the above consideration is the influence of the small ions on the concentration dependence of the collective diffusion coefficient. Taking into consideration the Belloni and Drifford model (15), we can reformulate Eqs. [2.34] and [2.42] in order to include the correction stemming from the finite diffusivity of the small ions:

$$
\begin{aligned}
& \lambda=1.45+\frac{\left(z_{0} e\right)^{2}}{\epsilon K T a} \frac{1}{(1+\kappa a)} \frac{3}{(\kappa a)^{2}} \\
& +\Delta \lambda_{\mathrm{SI}} \\
& \lambda=1.45+\frac{\Psi_{\mathrm{S}}^{2} \epsilon a}{K T}(1+\kappa a) \frac{3}{(\kappa a)^{2}} \\
& +\Delta \lambda_{\mathrm{SI}} \text {. }
\end{aligned}
$$

With $\Delta \lambda_{\text {sI }}$ we denoted the small ions correction (15). In the terms of our model it reads,

$$
\Delta \lambda_{\mathrm{SI}}=\frac{\left(z_{0} e\right)^{2}}{\epsilon K T a} \frac{1}{(1+\kappa a)^{2}} \frac{3}{(\kappa a)^{2}} \frac{D_{0}}{D_{\mathrm{SI}}},
$$

where $D_{\mathrm{SI}}$ is the small ions diffusion coefficient. We can estimate the relative contribution $\Delta \lambda_{\mathrm{SI}}$ using

$$
\frac{\Delta \lambda_{\mathrm{SI}}}{\lambda^{\mathrm{EL}}}=\frac{1}{(1+\kappa a)} \frac{D_{0}}{D_{\mathrm{SI}}} .
$$

The importance of $\Delta \lambda_{\mathrm{SI}}$ decays monotonically with $\kappa a$. Furthermore, it is proportional to the ratio $D_{0} / D_{\mathrm{SI}}$. Hence, for most particular cases (latex particles, ionic micelles, microemulsions) the correction $\Delta \lambda_{\mathrm{SI}}$ is less than $10 \%$.

At the end of this section we compare our results for the hydrodynamic terms (Eqs. [2.21], [2.25], [2.31]) to those of Phillies (12, 13) who accounted for the hydrodynamic interactions in a somewhat different way. As Phillies pointed out, the difference between his approach and the approach of Felderhof for 
charged particles is most pronounced for large $\kappa a$. For $\kappa a<2$ this difference is less than $10 \%$.

\section{LIGHT SCATTERING EXPERIMENTS}

For experimental verification of the theoretical model formulated above, we performed dynamic light scattering measurements (DLS) of the collective diffusion coefficient in latex suspensions at different volume fractions of particles and various concentrations of electrolyte.

\subsection{Materials and Sample Preparation}

The polystyrene latex suspension was commercially supplied by Interfacial Dynamics Corporation. According to the accompanying certificate, the diameter of the particles is $d=$ $19 \mathrm{~nm} \pm 20 \%$, the number of ionizable sulfonic groups per particle is $z=400$, and the solid content is $6.29 \mathrm{wt} \%$. The $\mathrm{NaCl}$ (Merck), used as electrolyte, was heated for $6 \mathrm{~h}$ at $600^{\circ} \mathrm{C}$ for removal of organic contaminants and humidity. The water was purified by a Milli-Q Organex system (Millipore).

The residual electrolyte, present in the commercial latex suspension, was removed by multiple consecutive dilutions with deionized water, and concentrations by means of membrane filtration. The filtration procedure was performed in a stirred batch cell at low pressure, $50 \mathrm{kPa}$, and moderate stirring. The membrane, we used, was polysulfonic with $10^{5}$ Daltons molecular weight cut off. The conductivity of the permeate was periodically measured and after achievement of constant value (about $0.3 \mathrm{mS} / \mathrm{m}$ ) the suspension was finally filtered through a $0.22-\mu \mathrm{m}$ filter unit (Millipore) and diluted to $1.00 \mathrm{wt} \%$. The concentration of latex was determined by drying and weighing a given volume of the suspension. In order to check whether some coagulation had occurred during the procedures described above, we compared the pretreated suspension with the initial one by means of DLS. The measurements were performed in excess of $\mathrm{NaCl}$ in order to avoid any electro- static and interaction effects. The measured mean diffusion coefficients and polydispersities were identical for both samples.

Suspensions of different concentrations of latex particles and $\mathrm{NaCl}$ were prepared without any further filtration, in order to avoid changes in the amounts of solutes.

\subsection{Light Scattering Apparatus}

The measurements were performed on Malvern 4700 C system, supplied with K7032 CE 8-Multibit correlator. The light source was an Argon laser (Innova 70, Coherent) operating at $488 \mathrm{~nm}$ wavelength of vertically plane polarized light. The scattering angle $\theta$ was varied from $10^{\circ}$ to $135^{\circ}$. This range of angles corresponds to a variation in the magnitude of the scattering vector $q=(4 \pi n / \Lambda) \sin (\theta / 2)$ between $3.00 \times 10^{4} \mathrm{~cm}^{-1}$ and $3.17 \times 10^{5}$ $\mathrm{cm}^{-1} . \Lambda$ is the wavelength of the light in vacuo and $n$ is the refractive index of the suspension. The temperature of the samples was automatically kept $25 \pm 0.1^{\circ} \mathrm{C}$.

The concentration of latex particles varied from 0.01 to $0.20 \mathrm{wt} \%$. Such values are high enough to allow measurements at minimum laser power (about $15 \mathrm{~mW}$ ). Thus, the results are not influenced by local heating of the samples caused by the beam. On the other hand, these concentrations are low enough to avoid multiple light scattering. In all cases the beam passing through the suspensions was clear, without a halo of secondary scattered light. The theoretical estimations also show that the multiple scattering is negligible for such small particles, at concentration below $0.3 \mathrm{wt} \%$; see Ref. (2), p. 129.

\subsection{Data Analysis}

Malvern $4700 \mathrm{C}$ works in the homodyne mode of operation. Hence, the photomultiplier detects the light intensity autocorrelation function,

$$
\begin{aligned}
g^{(2)}(\tau) & =\left\langle E_{\mathrm{S}}^{*}(t) E_{\mathrm{S}}(t)\right. \\
\times & \left.E_{\mathrm{S}}^{*}(t+\tau) E_{\mathrm{S}}(t+\tau)\right\rangle /\langle I\rangle^{2}
\end{aligned}
$$


where $E_{\mathrm{S}}$ is the scattered electric field, $I$ is the intensity of the scattered light, the angular brackets denote time averaging, and the asterisks denote complex conjugation. For relatively free diffusing scatterers (as in our case), $g^{(2)}(\tau)$ is related to the field autocorrelation function $g^{(1)}(\tau)$ via the Siegert relation (see, e.g., Ref. (2))

$$
g^{(2)}(\tau)=1+\left|g^{(1)}(\tau)\right|^{2} .
$$

By definition

$$
g^{(1)}(\tau)=\left\langle E_{\mathrm{S}}^{*}(t+\tau) E_{\mathrm{S}}(t)\right\rangle /\langle I\rangle,
$$

and for noninteracting monodisperse particles is represented by a single exponential function.

Hence, from the measured quantity $g^{(2)}(\tau)$ we can calculate the field autocorrelation function $g^{(1)}(\tau)$. A typical plot of the latter is shown in Fig. 2 for concentration of latex 0.04 wt $\%$ in $10^{-4} \mathrm{~mol} / 1 \mathrm{NaCl}$ at scattering angle $\theta$ $=30^{\circ}$. The logarithm of the correlation function $\ln g^{(1)}(\tau)$ vs $\tau$ significantly deviates from straight line at long times. This deviation is due (2) to: (i) the electrostatic and hydrodynamic interactions between the particles; (ii) the polydispersity of the particles. The detailed analysis of such complex correlation functions is a difficult and not entirely resolved problem. Nevertheless, some valuable information can be extracted from the initial decay of the autocorrelation function. A convenient analysis

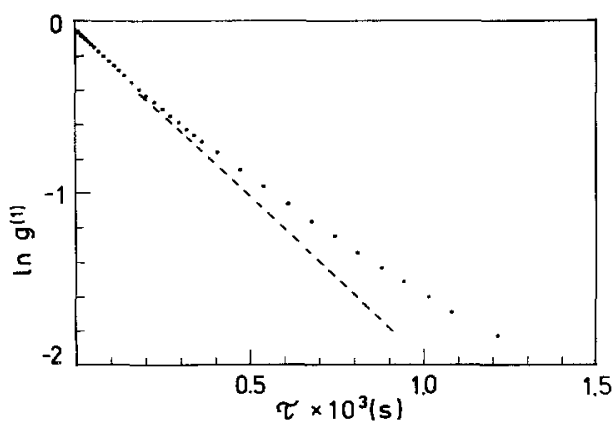

FIG. 2. Logarithm of the field autocorrelation function $g^{(1)}(q, \tau)$ vs $\tau$ at $0.04 \mathrm{wt} \%$ latex with $10^{-4} \mathrm{~mol} / 1 \mathrm{NaCl}$, at $q=8.90 \times 10^{4} \mathrm{~cm}^{-1}$. can be performed in terms of the cumulant expansion (2)

$$
\ln g^{(1)}(\tau)=\sum_{n} K_{n} \frac{(-\tau)^{n}}{n !},
$$

where the $n$th cumulant is determined as

$$
K_{n}=(-1)^{n} \lim _{\tau \rightarrow 0} \frac{d^{n}}{d \tau^{n}} \ln g^{(1)}(\tau) .
$$

For a system of noninteracting particles (2)

$$
K_{1}=D_{\mathrm{SE}} q^{2}, \quad \mathrm{~K}_{2}=\mathrm{K}_{3}=\cdots=0 .
$$

Hence the first cumulant is related to the Stokes-Einstein diffusion coefficient $D_{\mathrm{SE}}$. For a system of interacting particles, the first cumulant defines an effective diffusion coefficient $(2,16)$ :

$$
D_{\mathrm{EFF}}=K_{1} / q^{2} .
$$

The value of $D_{\mathrm{EFF}}$ depends on the scattering vector $q$ and the volume fraction of particles $\phi$. Relations expressing $D_{\mathrm{EFF}}$ as a function of $q$ and $\phi$ at low volume fractions and arbitrary scattering angles were given by several authors $(2,16)$. As known, in the small $q$-limit, $D_{\mathrm{EFF}}$ coincides with the collective diffusion coefficient $D_{\mathrm{C}}$ defined by Eq. [2.6]:

$$
D_{\mathrm{C}}(\phi)=\lim _{q \rightarrow 0}\left[K_{1}(q, \phi) / q^{2}\right] .
$$

Hence $D_{\mathrm{C}}$ can be determined by extrapolating the experimental curve $D_{\mathrm{EFF}}(q)$ for $\mathrm{q} \rightarrow 0 . D_{\mathrm{C}}$ thus determined is to be compared to the theoretical value, calculated by means of Eqs. [2.6], [2.34], and [2.42].

The experimental data were obtained and treated in the following manner:

(i) for given concentrations of latex particles and electrolyte we measured the autocorrelation function $g^{(1)}(\tau)$ at different scattering angles. Using the first cumulant we calculated the effective diffusion coefficient $D_{\mathrm{EFF}}(q, \phi)$ from each $g^{(1)}(\tau)$ curve;

(ii) after extrapolation of the plot of $D_{\mathrm{EFF}}$ vs $q$ for $q \rightarrow 0$ (see below for details), the collective diffusion coefficient $D_{\mathrm{C}}(\phi)$ was determined; 
(iii) in accordance with Eq. [2.6], the slope of the plot of $D_{\mathrm{C}}$ vs $\phi$ determines the interaction coefficient $\lambda$ at given concentration of low molecular electrolyte (i.e., at given value of $\kappa a$ ).

\section{RESULTS AND DISCUSSION}

The experimentally determined dependence of $D_{\mathrm{EFF}}$ on the scattering vector magnitude $q$ is shown in Fig. 3 for four different volume fractions of particles and concentration of electrolyte equal to $10^{-4} \mathrm{~mol} / 1 \mathrm{NaCl}$. The lines are drawn through the experimental points as described below. One sees that $D_{\text {EFF }}$ gradually increases with the decrease of $q$ (for given $\phi$ ), and tends to constant value at low scattering angles. We need the limit values $D_{\mathrm{C}}=D_{\mathrm{EFF}}(q$ $\rightarrow 0$ ), and for that purpose a numerical extrapolation of the experimental data was performed. The power expansion of the expression for the angular dependence of $D_{\mathrm{EFF}}$ for small $q$, given in Ref. (2), contains only even powers of $q$. A detailed analysis of the dependence $D_{\mathrm{EFF}}(q)$ is performed in Ref. (26). It

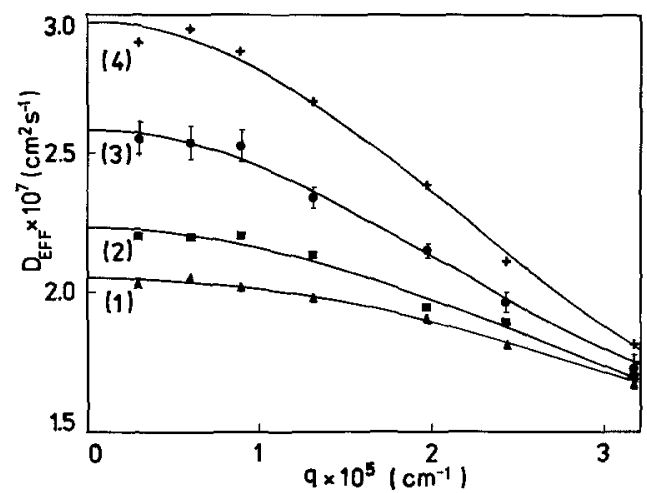

FIG. 3. Plot of $D_{\mathrm{EFF}}$ vs $q$ at $k a=0.422$ and for four different particle volume fractions. The experimental points are fitted with smooth curves, according to Eq. [4.1]: (1) $\phi=1 \times 10^{-4}, A=-3.94 \times 10^{-19} \mathrm{~cm}^{4} / \mathrm{s}, B=1.39$ $\times 10^{-31} \mathrm{~cm}^{6} / \mathrm{s}$; (2) $\phi=2 \times 10^{-4}, A=-7.37 \times 10^{-19}$ $\mathrm{cm}^{6} / \mathrm{s}, B=1.97 \times 10^{-30} \mathrm{~cm}^{6} / \mathrm{s} ;(3) \phi=4 \times 10^{-4}, A=$ $-13.9 \times 10^{-19} \mathrm{~cm}^{4} / \mathrm{s}, B=5.06 \times 10^{-30} \mathrm{~cm}^{6} / \mathrm{s}$; (4) $\phi=$ $6 \times 10^{-4}, A=-17.8 \times 10^{-19} \mathrm{~cm}^{6} / \mathrm{s}, B=6.19 \times 10^{-30}$ $\mathrm{cm}^{6} / \mathrm{s}$. The values of $D_{\mathrm{C}}$ are shown in Fig. 4 . was shown that a polynomial least square fit can be used:

$$
D_{\mathrm{EFF}}(q)=D_{\mathrm{C}}+A q^{2}+B q^{4} .
$$

The coefficients $D_{\mathrm{C}}, A$, and $B$ are to be determined by means of the least square method. The respective curves are shown in Fig. 3. The experimental points are randomly distributed with respect to the smooth curves, and the relative errors are less than $5 \%$. Thus, we determined four values of $D_{\mathrm{C}}$ corresponding to different particle volume fractions; see line 1 in Fig. 4. The electrolyte present in the suspension was $10^{-4} \mathrm{~mol} / 1 \mathrm{NaCl}$. The higher volume fraction $\phi$, the higher collective diffusion coefficient $D_{\mathrm{C}}$. The lines in Fig. 4 are fitted according to the equation

$$
D_{\mathrm{C}}(\phi)=D_{0}(1+\lambda \phi) .
$$

The intersection gives the diffusion coefficient of a single particle at infinite dilution of the latex spheres. It can depend on the electrolyte type and concentration (19). The other lines plotted in Fig. 4 correspond to $2.25 \times 10^{-4}$, $9.00 \times 10^{-4}$, and $10^{-2} \mathrm{~mol} / 1 \mathrm{NaCl}$, respectively. They were obtained by following the same procedure as for line 1 .

A substantial feature of the results presented in Fig. 4 is that the intersection (the single particle diffusion coefficient $D_{0}$ ) depends on the electrolyte concentration. This is a known electrokinetic effect, which was investigated both theoretically and experimentally by several authors (17-19). They found that $D_{0}$ passes through a minimum at $\kappa a \approx 1$. At $\kappa a$ $\geqslant 3$, Schumacher and Van den Ven (19) showed experimentally and theoretically that $D_{0}$ coincides with the Stokes-Einstein diffusion coefficient $D_{\text {SE }}$. In our case, $\kappa a \approx 3$ is reached at $10^{-2} \mathrm{~mol} / \mathrm{l}$ concentration of electrolyte, so we supposed that the intersection of line 4 in Fig. 4 gives the value $D_{0}=D_{\mathrm{SE}}$. This allowed us to find the mean hydrodynamic radius of the latex particles $a=12.7$ $\mathrm{nm}$. Figure 5 presents the plot of the ratio $D_{0} /$ $D_{\mathrm{SE}}$ vs $\kappa a$. The curve shown there is similar 


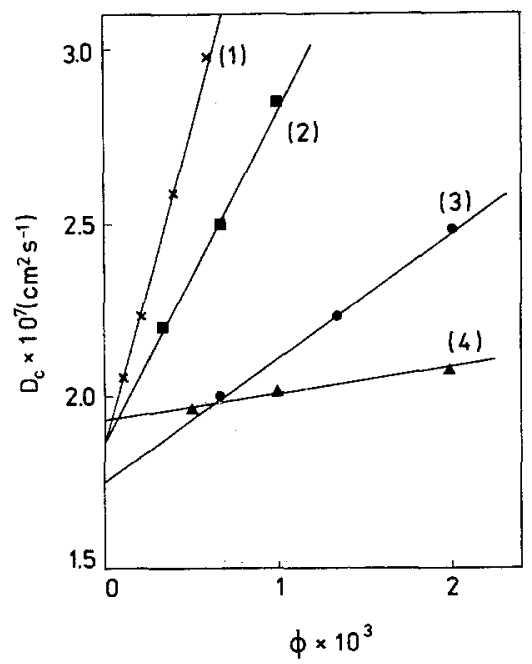

FIG. 4. Plot of $D_{\mathrm{C}}$ as a function of the volume fraction of latex particles $\phi$ at different electrolyte concentrations: (1),$\kappa a=0.422 ;(2), \kappa a=0.632 ;(3), \kappa a=1.27 ;(4), \kappa a$ $=4.22$.

to that obtained from Schumacher and Van den Ven (19) (see their Fig. 7) for constant surface potential. The theoretical model given by Schurr (17) and Medina-Noyola and Vizcarra-Rendon (18) predicts similar behavior, but at constant surface charge. If the surface potential is kept constant, this theory $(17,18)$ predicts monotonous decrease of the single particle diffusion coefficient with $\kappa a$. Since the theories (17-19) are known to be only in qualitative agreement with the experiments, we did not apply them to interpret quantitatively the results shown in Fig. 5.

Figure 6 shows the dependence of the interaction coefficient $\lambda$ (determined from the slope of the lines in Fig. 4) on the electrolyte concentration $\kappa a$. The points represent our experimental results, whereas the smooth line is drawn according to Eq. [2.42] at constant surface potential $\Psi_{\mathrm{S}}=37.7 \mathrm{mV}$. The dashed line calculated by means of Eq. [2.40] shows the dependence of the particle charge number $z_{0}$ on $\kappa a$. These results demonstrate that the constant potential hypothesis agrees much better with our data than the constant charge hypothesis. The particle charge $z_{0}$ strongly increases with the increase of the electrolyte concentration (see also Table I). This means that the degree of dissociation of the surface ionizable groups also increases from $10 \%$ at $\kappa a=0.422$ to $38 \%$ at $\kappa a=4.22$. The value of the surface potential, $e \Psi_{\mathrm{S}} / K T \approx 1.5$, is too high for the linear theory to be strictly valid, but still it can be applied as demonstrated in Ref. (27).

Our theoretical expression for $\lambda$ can be also checked by using the experimental results of Anderson et al. (11) for the diffusion coefficient of bovine serum albumin at low ionic strengths. The points in Fig. 7 are the data of Anderson et al., whereas the smooth line is drawn in accordance with formula [2.34]. Curve 2 in Fig. 7 represents the independently determined data for the particle charge $z_{0}$ given in Ref. (8), which were used to calculate the theoretical curve 1 in Fig. 7; see also Table II. Hence, $z_{0}$ was not an adjustable parameter in this case. The agreement between our model (Eq. [2.34]) and the experiment of Anderson et al. (11) seems to be very good.

By the end of this section we discuss three additional problems, arising from the particular system under consideration:

(i) The real latex suspension is not perfectly monodisperse, and in fact the measured values of $D_{\mathrm{EFF}}$ are averaged over the particle size distribution. We believe this is the main reason for the difference between the hydrodynamic radius $a=12.7 \mathrm{~nm}$, obtained by DLS, and

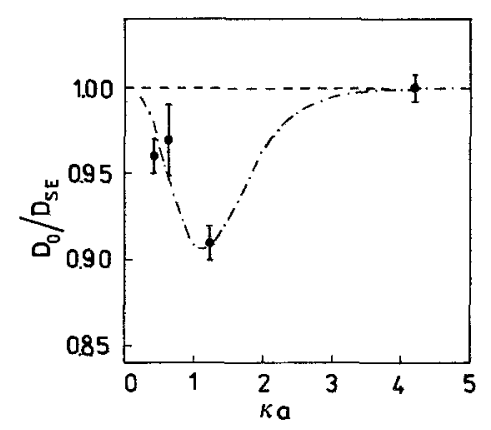

FIG. 5. Plot of $D_{0} / D_{\text {SE }}$ vs $\kappa a$. 


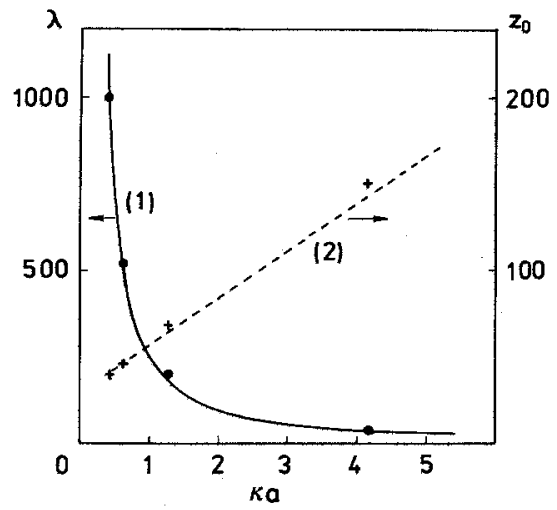

Fig. 6. Plot of the interaction parameter $\lambda$, and the particle charge $z_{0}$ vs $\kappa a$ for latex. The curves (1) and (2) are drawn through the experimental points at constant surface potential $\Psi_{\mathrm{s}}=37.7 \mathrm{mV}$ by means of Eqs. [2.42] and $[2.40]$, respectively.

the certified one, $a=9.5 \mathrm{~nm}$. The latter value was probably obtained by electron microscopy and was a number averaged quantity, while the former was averaged over the intensity of the scattered light. This is known as intensityaveraging (2) and should lead to larger values for such small particles;

(ii) As we already mentioned in Section 2, the screening parameter $\kappa$ in the theory of Beresford-Smith et al. (22) includes both the added electrolyte and counterions dissociated from the colloidal particles. The numerical estimations showed that for the particle and electrolyte concentrations we used, the effect of the dissociated counterions on $\kappa$ is less than $5 \%$, so their presence can be neglected in this case. (iii) The linearization of the correlation function (see Eqs. [2.13] and [2.14]) also needs some discussion. This approximation is applicable for colloidal systems of small and weakly charged particles such as proteins and some ionic micelles. Hence, we can expect that the application of our theoretical model to the results of Anderson et al. (11) is quite reasonable. For latex particles, however, the situation is different. Usually they have greater density of the surface charge, compared to proteins. Hence, the linearization of the correlation function can be applied only for larger interparticle distances, where the interaction energy has already sufficiently decreased, due to its specific form (Eq. [2.14]). Still, we cannot claim that the particle charge of the latex particles calculated by us and shown with curve 2 in Fig. 7 is the real one. Most probably it is an effective value. A rigorous procedure for treatment of the diffusion in dilute suspensions of strongly charged particles was developed recently (26). By applying the Barker and Henderson perturbation theory (28), and involving the nonlinear Poisson-Boltzmann equation, a straightforward relation between the actual particle charge (or potential) and the correction parameter $\lambda$ was obtained.

\section{CONCLUDING REMARKS}

We derived an expression for the electrostatic contribution $\lambda^{\mathrm{EL}}$ to the interaction parameter $\lambda$. The latter characterizes the linear dependence of the collective diffusion coeffi-

TABLE I

Parameters of the Latex Suspension Used in the Dynamic Light Scattering Experiments

\begin{tabular}{rcrrrr}
\hline $\mathrm{NaCl}(\mathrm{mol} / 1)$ & $\kappa a$ & $D_{0} \times 10^{7}\left(\mathrm{~cm}^{2} / \mathrm{s}\right)$ & \multicolumn{1}{c}{$\lambda$} & \multicolumn{1}{c}{$\frac{e \Psi_{s}{ }^{a}}{K T}$} & $z_{0}{ }^{b}$ \\
\hline $1.0 \times 10^{-4}$ & 0.422 & $1.86 \pm 0.01$ & $1000 \pm 20$ & $1.51 \pm 0.02$ & $39.3 \pm 0.4$ \\
$2.25 \times 10^{-4}$ & 0.632 & $1.86 \pm 0.03$ & $520 \pm 20$ & $1.52 \pm 0.05$ & $45.6 \pm 2.0$ \\
$9.0 \times 10^{-4}$ & 1.270 & $1.75 \pm 0.02$ & $206 \pm 6$ & $1.63 \pm 0.05$ & $68.0 \pm 2.0$ \\
$1.0 \times 10^{-2}$ & 4.220 & $1.93 \pm 0.02$ & $40 \pm 8$ & $1.57 \pm 0.20$ & $150.0 \pm 15.0$ \\
\hline
\end{tabular}

${ }^{a}$ Dimensionless potential, calculated in the framework of the linear theory (see Eq. [2.42]).

${ }^{b}$ Surface charge, determined from the interaction parameter (see Eq. [2.34]). 


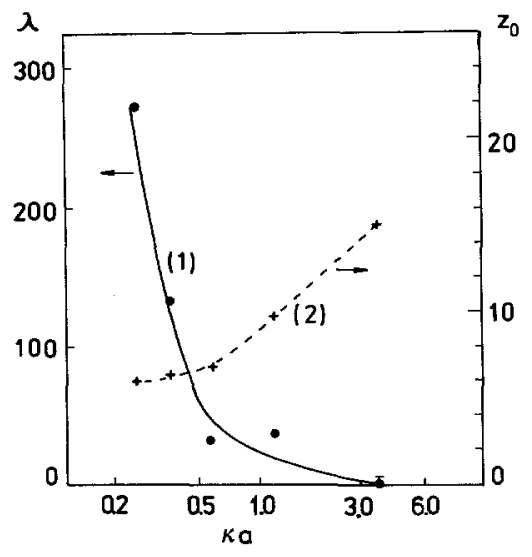

FIG. 7. Check of our Eq. [2.34] (Curve 1) against the experimental data for $\lambda(\bullet)$, and $z_{0}(+)$, taken from Ref. (11). The data for $z_{0}$ are used to calculate curve 1 without any adjustable parameter.

cient $D_{\mathrm{C}}$ on the particle volume fraction $\phi$ (see Eq. [2.6]). It was shown that the contributions of the short range hydrodynamic interactions $\lambda_{\mathrm{A}}^{\mathrm{EL}}$ and $\lambda_{\mathrm{S}}^{\mathrm{EL}}$ cannot exceed $3 \%$ of $\lambda^{\mathrm{EL}}$ for any electrolyte concentration; see Fig. 1. Hence, in fact $\lambda^{\mathrm{EL}}$ can be expressed as a sum of the osmotic virial term $\lambda_{\mathrm{V}}^{\mathrm{EL}}$ and the long range hydrodynamic Oseen term $\lambda_{\mathrm{O}}^{\mathrm{EL}}$. The result is a simple formula-Eq. [2.34] or the equivalent Eq. [2.42]. The theoretical model predicts large values of the interaction parameter $\lambda$ (about several hundreds) at low concentrations of electrolyte.

Light scattering experimental investigations of well defined latex suspensions were performed in order to obtain the dependence of the effective diffusion coefficient $D_{\mathrm{EFF}}$ on the scattering vector $q$, and the particle volume fraction $\phi$ at different electrolyte concentrations (see Fig. 3). Using an adequate numerical extrapolation of the function $D_{\mathrm{EFF}}(q)$ we obtained the dependence of the collective diffusion coefficient $D_{\mathrm{C}}$ on the volume fraction and electrolyte concentration (see Fig. 4). A value $\lambda=1000$ was reached at low electrolyte concentrations (cf., Fig. 6). The experimental points in Fig. 6 are in excellent agreement with the theoretical model Eq. [2.42] at constant surface potential $\Psi_{\mathrm{S}}=37.7 \mathrm{mV}$ (curve 1).
The particle charge (calculated from the experimental values of $\lambda^{\mathrm{EL}}$ ) increases with $\kappa a$ (curve 2 in Fig. 6).

The experimental data of Anderson et al. (11), for bovine serum albumin, are treated in a different manner. In this case the theoretical curve (Fig. 7) was drawn without any adjustable parameter because independently measured values of the surface charge of the protein molecule were available in Ref. (11); see also Table II. The agreement between the theory and experiment turns out to be very good (see Curve 1 in Fig. 7). In this case, the surface charge increases several times with $\kappa a$, while the potential $\Psi_{\mathrm{S}}$ changes only within $30 \%$.

It was found that the values for the collective diffusion coefficient $D_{\mathrm{C}}$ taken at $\phi=0$ could differ for different electrolyte concentrations, passing through a minimum at $\kappa a \approx 1$ (Fig. 5 ). This result is in agreement with the theoretical and experimental investigations of Schumacher and Van den Ven (19) concerning the influence of the electric double layer on the single particle diffusion.

The effect of the electrostatic pair interactions on the short time self diffusion coefficient (see Eq. [2.25], combined with Eq. [2.35]) and the sedimentation velocity (Eq. [2.37]) was also considered.

\section{TABLE II}

Parameters of the Bovine Serum Albumin Solutions used by Anderson et al. (11)

\begin{tabular}{lrrrrr}
\hline $\mathrm{KCl}(\mathrm{mol} / \mathrm{l})$ & $\kappa a$ & $\lambda^{a}$ & \multicolumn{1}{c}{$\lambda^{b}$} & \multicolumn{1}{c}{$z_{\mathrm{o}}{ }^{c}$} & $\frac{e \Psi_{\mathrm{s}}{ }^{\mathrm{c}}}{K T}$ \\
\hline $5.0 \times 10^{-4}$ & 0.27 & 224 & 272 & 6.0 & 0.93 \\
$1.0 \times 10^{-3}$ & 0.37 & 122 & 133 & 6.3 & 0.90 \\
$2.5 \times 10^{-3}$ & 0.60 & 46 & 31 & 6.8 & 0.84 \\
$1.0 \times 10^{-2}$ & 1.20 & 17 & 38 & 9.6 & 0.86 \\
$1.0 \times 10^{-1}$ & 3.70 & 2 & 0 & 15.1 & 0.63 \\
\hline
\end{tabular}

${ }^{a}$ Calculated with the help of our model (Eq. [2.34]) and values of the parameter $z_{0}$ given by Anderson et al. (11).

${ }^{b}$ Experimental results for bovine serum albumin taken from Ref. (11).

${ }^{c}$ Values taken from Table II in Ref. (11). 
The results obtained in the present paper are applicable to a wide range of colloidal systems: suspensions, micellar, and microemulsion solutions. They may render particular importance in studying solutions of globular proteins which have relatively low surface charge, and in this case, the assumptions implied in our model are mostly justified.

The proposed model (Eqs. [2.34] and [2.42]) allows us to predict the diffusion coefficient if the colloidal particle size, charge (or potential), ion concentrations, and other parameters are known. On the other hand, the equations derived can be used to determine some of these parameters (e.g., surface charge or potential) by measuring the diffusion coefficient via light scattering or some other convenient method.

\section{APPENDIX}

DERIVATION OF ASYMPTOTIC EXPRESSIONS FOR $\lambda_{A}^{E L}$ AND $\lambda_{S}^{E L}$

The problems for the explicit calculation of $\lambda_{A}^{E L}$ and $\lambda_{S}^{E L}$ arise from the appearance of integral exponents of different order $n$ :

$$
E_{n}(p)=\int_{1}^{\infty} \frac{e^{-s p}}{s^{n}} \mathrm{ds},
$$

$$
n=1,2,3, \ldots .
$$

Every integral exponent of higher order can be reduced to an expression, depending on $E_{1}(p)$, using the formula (23)

$$
\begin{aligned}
& \int \frac{e^{\beta x}}{x^{n}} d x=-\frac{e^{\beta x}}{(n-1) x^{n-1}} \\
& -\frac{\beta e^{\beta x}}{(n-1)(n-2) x^{n-2}} \\
& -\cdots-\frac{\beta^{n-2} e^{\beta x}}{(n-1) ! x}+\frac{\beta^{n-1}}{(n-1) !} E_{1}(p)
\end{aligned}
$$

for $n>1$.

Hence the mathematical difficulties are focused in the integral $E_{1}(p)$.

\section{A.1. Asymptotic Solutions for $\kappa a \ll 1$}

We can write (24)

$$
\begin{aligned}
& E_{1}(p) \\
& \quad=-C-\ln (p)-\sum_{n=1}(-1)^{n} \frac{(p)^{n}}{n n !},
\end{aligned}
$$

where $C=0.5772$ is the Euler constant. For $p=2 \kappa a \ll 1$ one obtains

$$
E_{1}(2 \kappa a) \approx-C-\ln (2 \kappa a) .
$$

In all expressions of interest, (see Eqs. [2.25], [2.31], and [A.2]), $E_{1}(2 \kappa a)$ is multiplied by a factor of the type $(2 \kappa a)^{n}$, where $n>2$. Using the l'Hôpital rule, it can be shown that

$$
\lim _{2 \kappa a \rightarrow 0}\left[(2 \kappa a)^{n} \ln (2 \kappa a)\right]=0 .
$$

Using Eqs. [A.2], [A.4], and [A.5], and considering terms linear with respect to $2 \kappa a$, we can write

$$
\begin{aligned}
& E_{n}(2 \kappa a) \\
& \quad \approx \frac{e^{-2 \kappa a}}{(n-1)(2 \kappa a)}\left(1-\frac{2 \kappa a}{(n-2)}\right) .
\end{aligned}
$$

Equation [A.6] can be introduced in the expressions [2.25] and [2.31] for estimating the terms $\lambda_{\mathrm{A}}^{\mathrm{EL}}$ and $\lambda_{\mathrm{S}}^{\mathrm{EL}}$ when $\kappa a \ll 1$.

\section{A.2. Asymptotic Solutions for $\kappa a \gg 1$}

In this limiting case we have $(23,24)$

$$
\begin{aligned}
& E_{n}(p) \\
& =\frac{e^{-p}}{p}\left(1-\frac{n}{p}+\frac{n(n+1)}{p^{2}}-\cdots\right) .
\end{aligned}
$$

Hence

$$
E_{n}(2 \kappa a) \approx \frac{e^{-2 \kappa a}}{2 \kappa a}\left(1-\frac{n}{2 \kappa a}\right) .
$$

The sum in the brackets is restricted to the term linear with respect to $(2 k a)^{-1}$. Introducing Eq. [A.8] in the expressions [2.25] and [2.31], we obtain asymptotic expressions for $\lambda_{\mathrm{A}}^{\mathrm{EL}}$ and $\lambda_{\mathrm{S}}^{\mathrm{EL}}$, this time for $\kappa a \gg 1$. 


\section{ACKNOWLEDGMENTS}

We are indebted to Prof. B. U. Felderhof for reading the first version of the manuscript and making some critical remarks, to Dr. P. A. Kralchevsky for the helpful discussions, and to Mrs. R. Alargova for drawing the figures. This work was supported financially by the Bulgarian Ministry of Science and Higher Education.

\section{REFERENCES}

1. Einstein, A., Ann. Phys. (Leipzig) 17, 549 (1905).

2. Pusey, P. N., and Tough, R. J. A., in "Dynamic Light Scattering" (R. Pecora, Ed.), Chap. 2. Plenum Press, New York, 1985.

3. Batchelor, G. K., J. Fluid Mech. 52, 245 (1972).

4. Batchelor, G. K., J. Fluid Mech. 74, 1 (1976).

5. Felderhof, B. U., J. Phys. A: Math. Gen. 11, 929 (1978).

6. Felderhof, B. U., Physica A: 89, 373 (1977).

7. Jones, R. B., and Schmitz, R., Physica $A:$ 149, 373 (1988).

8. Cichocki, B., and Felderhof, B. U., J. Chem. Phys. 89, 1049 (1988).

9. Cichocki, B., Felderhof, B. U., and Schmitz, R., PCH, PhysicoChem. Hydrodyn. 10, 383 (1988).

10. Anderson, J. L., and Reed, C. C., J. Chem. Phys. 64, 3240 (1976).

11. Anderson, J. L., Rauh, F., and Morales, A., J. Phys. Chem. 82, 608 (1978).

12. Phillies, G. D. J., J. Chem. Phys. 77, 2623 (1982).
13. Phillies, G. D. J., J. Colloid Interface Sci. 119, 518 (1987).

14. Ohtsuki, T., and Okano, K., J. Chem. Phys. 77, 1443 (1982).

15. Belloni, L., and Drifford, M., J. Phys. Lett. 46, 1183 (1985).

16. Cichocki, B., and Felderhof, B. U., J. Chem. Phys. 94, 556 (1991).

17. Schurr, J. M., Chem. Phys, 45, 119 (1980).

18. Medina-Noyola, M., and Vizcarra-Rendon, A., Phys. Rev. A: 32, 3596 (1985).

19. Schumacher, G. A., and Van den Ven, T. G. M., Faraday Discuss. Chem. Soc. 83, 75 (1987).

20. Russel, W. B., J. Colloid Interface Sci. 55, 590 (1976).

21. McQuarrie, D. A., "Statistical Mechanics," Ch. 15. Harper \& Row, New York, 1976.

22. Beresford-Smith, B., Chan, D. Y. C., and Mitchell, D. J., J. Colloid Interface Sci. 105, 216 (1985).

23. Dwight, H. B., "Tables of Integrals." Macmillan, New York, 1961.

24. Abramowitz, M., and Stegun, I. A., "Handbook of Mathematical Functions." National Bureau of Standards, 1964.

25. Corti, M., and Degiorgio, V., J. Phys. Chem. 85, 711 (1981).

26. Denkov, N. D., and Petsev, D. N., Physica A, in press.

27. Overbeek, J.Th.G., in "Colloid Science" (H. R. Kruyt Ed.), Chap. 4. Elsevier, Amsterdam, 1952.

28. Barker, J. A., and Henderson, D., Rev. Mod. Phys. 48, 587 (1976). 